\title{
Mitral valvar prolapse: overlap or masked syndrome?
}

\author{
Luciano Daliento \\ Department of Cardiology, University of Padua Medical School, Padua, Italy
}

1 HE PREVALENCE OF PROLAPSE OF THE MITRAL valve in the general population is estimated to be approximately $4-5 \% .^{1}$ Even if this condition is benign, it produces anxiety in many patients, as well as in many doctors, because of the potential risk of severe complications, such as infective endocarditis, surgical indications for valvar incompetence, cerebral ischemia, and particularly syncope or sudden death.

In recent years, several investigators have tried to obtain a more accurate stratification of risk in patients with mitral valvar prolapse. Besides more traditional techniques, advanced methods were adopted, including signal-averaging electrocardiography, analysis of variability in heart rate and measurement of QT dispersion. ${ }^{2,3}$ All of them provided useful information to evaluate the prognosis of these patients. In fact reduced variability in heart rate, increased QT dispersion, and particularly presence of late potentials, are associated with an increased electrical instability. Late potentials are present also in children with mitral valvar prolapse and ventricular arrhythmias, as reported by Bobkowski and colleagues in this issue of Cardiology in the Young. ${ }^{4}$ Following their compulsive effort to define prognosis, however, we need to clarify some semantic problems related to mitral valvar prolapse.

In 1988, Quill' introduced the term "overlap syndromes" to indicate anatomico-physiologic phenomenons that largely overlap with normal ones. These entities, which had become labelled as disease processes by the medical community through a process of over-medicalization, included mitral valvar prolapse, hypoglycemia, irritable colon, and premenstrual syndrome. It is questionable if this pessimistic behaviour

\footnotetext{
Correspondence to: Luciano Daliento MD, FACC, FESC, Department of Cardiology, University of Padua Medical School, Padua, Italy. E-mail: luciano.daliento@ unipd.it

Accepted for publication 26 April 2002
}

is justified, considering that the risk of serious complications is very low. For syncope and sudden death, for example, complications at $0.1 \%$ per year do not significantly differ from those of $0.2 \%$ for the general population. ${ }^{6}$ It is certainly the case, however, that extensive use of the term "prolapse", after the introduction and diffusion of echocardiography, has increased the confusion. In the original description of Barlow and Pocock, ${ }^{7}$ prolapse as applied to the mitral valve always implied a pathological status. This was characterized at gross examination by thickened and larger leaflets, associated with elongated, tortuous, attenuated or thinned tendinous cords, or the presence of endocardial plaques associated with focal myocardial fibrosis. These lesions ${ }^{8}$ are the expression of myxoid degeneration of collagen and elastic fibers, with significant thickening of the spongy and fibrous layers of the leaflets, causing dysfunction of the overall valvar apparatus. This then functions as the substrate of the electrical instability which probably underscores sudden death. In fact, ventricular arrhythmias are more common in the subgroup of patients with severe mitral incompetence and left ventricular dysfunction, in whom the risk of sudden death becomes significant, reaching from 0.9 to $2 \%$.

In the new era of echocardiography, all patients with systolic bulging of the valvar leaflets into the left atrium, independent of the morphofunctional status of the valvar structures, are labelled as having prolapse. Furthermore, this subgroup of subjects, without evident signs of myxoid dystrophy, may be complicated by arrhythmias, and more rarely by sudden death. To detect criterions of the risk for syncope or sudden death in such patients without severe mitral dystrophy, and with few symptoms, is more difficult. It requires a different diagnostic approach. In the series of 163 cases of sudden cardiovascular death in young people reported by Corrado and colleagues, ${ }^{9} 17(10 \%)$ were found to have isolated prolapse of the mitral valve at gross autopsy 
examination. In only 8 patients had the prolapse been diagnosed during life. Of the other patients, 6 had suffered palpitations and/or syncope, while 3 had premature ventricular beats. Only an accurate histopathologic study of all cardiac structures clarified the diagnosis, and revealed the true substrate of the ventricular electrical instability. Thus, in 9 cases there was focal myocardial atrophy and fatty replacement of the right ventricular walls, particularly in the outflow tract. Left ventricular myocardial disarray without hypertrophy was seen in 2 , and lymphocytic infiltrates in one. Abnormalities of the specialized conduction system were evident in 2 further patients. This accurate histopathological analysis also revealed that more than three-quarters of cases of isolated mitral valvar prolapse showed a potentially arrhythmogenic myocardial substrate not related to the morphofunctional status of the mitral valve. In the majority of cases, it was related to structural modifications of the right parietal walls. In these cases, the possibility of a clinically correct diagnosis was restricted by the presence of an echocardiographic finding of systolic bulging of the valvar leaflets. Thus, a more detailed qualitative and quantitative examination of the cardiac parietal walls, and of the characteristics of electrical instability, including the morphology of ventricular arrhythmias, along with research of abnormalities of depolarization or repolarization, and phases at basic or amplified electrocardiography, was excluded. In fact, the presence of ventricular beats arising from the right ventricle with left bundle branch block morphology should encourage a more accurate evaluation of coexisting cardiomyopathy. In children and adolescents, in particular, this could be present in a concealed form. Late potentials are the representation of delayed conduction in myocardial zones with a slow or inhomogeneous pattern of activation. The anatomical substrate responsible for such late potentials could be normal muscle bundles uncoupled by intervening fibrous tissue, small zones of scarred myocardium with very slow conduction, or large myocardial zones with asynchronous activation. ${ }^{10}$ Late potentials cannot, however, be specific for arrhythmogenic tissue. We have to assume that their appearance reflects the presence of functional or anatomical altered myocardium. Consequently, the detection of late potentials in patients classified as having an isolated mitral valvar prolapse, as reported by Bobkowski and colleagues, ${ }^{4}$ should be considered as expressions of cardiac disease not confined to the mitral valve. Likewise, the occurrence of sustained ventricular tachycardia in these patients requires an accurate evaluation to exclude the presence of an underlying myocardial disease. In fact, in "true" idiopathic ventricular tachycardia without proven structural cardiac disease, the prevalence of late potentials does not exceed that observed in normal individuals, at 0 to $5 \%$. In "apparent" idiopathic disease, this rises to 20 to $40 \%$, reaching a maximum of 70 to $85 \%$ in patients with arrhythmogenic right ventricular cardiomyopathy. ${ }^{11}$

Areas with inhomogeneous activation may provide the substrate for ventricular arrhythmias based on a re-entrant mechanism. Thus, as documented in different studies, the most impressive findings compared the presence of late potentials with the occurrence of sustained or non-sustained ventricular tachycardia. ${ }^{12}$ In contrast, less significant correlations were found between the presence of late potentials and isolated ventricular beats, as well as with the occurrence of sudden death or ventricular fibrillation. ${ }^{13}$ The presence of late potentials also contributes to reducing the area of overlap syndrome, and clarifies the masked forms. In children, in particular, the masked forms most frequently represent evidence of the onset of structural myocardial abnormalities.

The importance of the contribution of studies designed to stratify risk is evident, and could reasonably be extended to evaluate prognostic factors as predictors of critical events. We must remember, however, that all the conclusions we draw on the results on our cohort of patients with mitral valvar prolapse must be applicable, and will be applicable, to all subjects with this diagnosis.

\section{References}

1. Bourdarias JP. Le prolapsus valvulaire mitral:une anomalie grave? Arch Mal Coeur Viss 1991; 84: 981-986.

2. Ulgen MS, Biyik I, Karadede A, Temamogullari AV, Alan S, Tofrak N. Relation between QT dispersion and ventricular arrhythmias in uncomplicated isolated mitral valve prolapse. Jpn Circ J 1999; 63: 929-933.

3. Leclerc JF, Malergue MC, Coumel P. Late potentials and mitral valve prolapse. Arch Mal Coeur Vaiss 1993; 86: 285-289.

4. Bobkowski W, Siwinska A, Zachwieja J, Mrozinski B, RzeznikBieniaszewska A, Maciejewski J. Significance of ventricular late potentials in children with mitral valve prolapse: a prospective study. Cardiol Young 2002; 12: 333-338.

5. Quill TE, Lipkin M, Greenland P. The medicatization of normal variants: the case of mitral valve prolapse. J Gen Intern Med 1988; 3: 267-276

6. Fauchier JP, Babuty D, Fauchier L, et al. Mitral valve prolapse, arrhythmias and sudden death. Arch Mal coeur Vaiss 2000; 93: 1541-1547.

7. Barlow JB, Pocock WA. Mitral valve billowing and prolapse: perspective at 25 years. Herz 1988; 13: 227-234.

8. Virmani R, Atkinson JB, Forman MB. The pathology of mitral valve prolapse. Herz 1988; 13: 215-226.

9. Corrado D, Basso C, Nava A, Rossi L, Thiene G. Sudden death in young people with apparently isolated mitral valve prolapse. G Ital Cardiol 1997; 27: 1097-1105.

10. El-Sherif N. Electrophysiologic basis of ventricular late potentials. Prog Cardiovasc Dis 1993; 35: 417-427. 
11. Fauchier JP, Fauchier L, Babuty D, Cosnay P. Time-domain signalaveraged electrocardiogram in non ischemic ventricular tachycardia. Pacing Clin Electrophysiol 1996; 19: 231-244.

12. Borgreefe M, Breithard G. Pathophysiological mechanisms and clinical significance of ventricular late potentials. Eur Heart J 1986; 7: 364-385.
13. Hood MA, Pogwizd SM, Peirick J, Cian ME. Contribution of myocardium responsible for ventricular tachycardia to abnormalities detected by analysis of signal-averaged ECGs. Circulation 1992; 86: 1888-1901. 